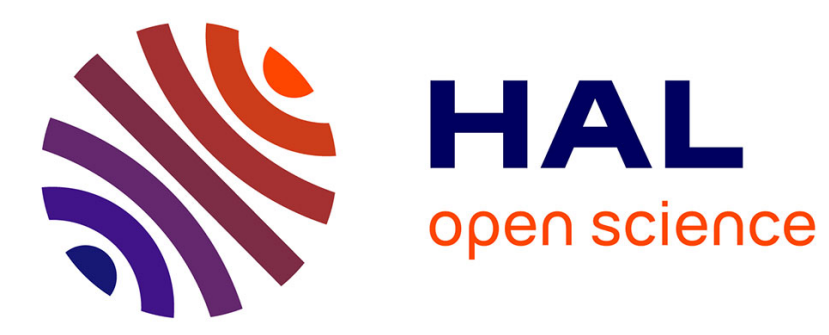

\title{
Effects of interaural differences on the loudness of low-frequency pure tones
}

\author{
Vincent Koehl, Mathieu Paquier, Etienne Hendrickx
}

\section{To cite this version:}

Vincent Koehl, Mathieu Paquier, Etienne Hendrickx. Effects of interaural differences on the loudness of low-frequency pure tones. Acta Acustica united with Acustica, 2015, 101 (6), pp.1168-1173. 10.3813/AAA.918909 . hal-01225306

\section{HAL Id: hal-01225306 https://hal.univ-brest.fr/hal-01225306}

Submitted on 10 Nov 2016

HAL is a multi-disciplinary open access archive for the deposit and dissemination of scientific research documents, whether they are published or not. The documents may come from teaching and research institutions in France or abroad, or from public or private research centers.
L'archive ouverte pluridisciplinaire HAL, est destinée au dépôt et à la diffusion de documents scientifiques de niveau recherche, publiés ou non, émanant des établissements d'enseignement et de recherche français ou étrangers, des laboratoires publics ou privés. 


\title{
Effects of interaural differences on the loudness of low-frequency pure tones
}

\author{
V. Koehl, M. Paquier, E. Hendrickx \\ Lab-STICC (UMR CNRS 6285), University of Brest, 6 avenue Victor le Gorgeu, 29200 Brest, France \\ vincent.koehl@univ-brest.fr
}

\begin{abstract}
Summary
The loudness related to a sound may vary according to the localization of its source. This phenomenon is described as directional loudness and has been mainly observed for high-frequency sounds and for sources located in the horizontal plane. Because of the acoustic shadow of the head, the left and right ear pressures are modified depending on the source azimuth and the global loudness resulting from a summation process may vary accordingly. But directional loudness has also been reported to occur at $400 \mathrm{~Hz}$, where shadowing effects are usually rather small. It might therefore be suspected that directional loudness effects could be influenced by other parameters involved in the localization process. In a previous study, a small but significant increase of loudness with increasing interaural time difference (ITD) was shown for low-frequency pure tones (200 and $400 \mathrm{~Hz}$ ) at a low loudness level (40 phon). The present study aimed at getting insight into the potential cause and extent of this effect by assessing the loudness of similar pure tones lateralized with headphones by applying an interaural level difference (ILD) in addition to an ITD and by measuring the effect of ITD at the hearing threshold. It showed significant effects of both ILD and ITD on loudness, and no interaction between these factors. As the effects added even when the factors were contradictory, it supports the hypothesis that the effect is caused by the ITD itself and is not related to the localization process. However, the ITD effect was not significant at the hearing threshold.
\end{abstract}

PACS no. 43.66. Cb, 43.66.Pn, 43.66.Rq

\section{Introduction}

Significant variations of loudness were observed when presenting bands of noise through loudspeakers that were located at various directions around a listener in an anechoic room [1, 2]. As an example, a third-octave noise band centered at $5 \mathrm{kHz}$ emitted by a lateral source $\left( \pm 90^{\circ}\right)$ was perceived as louder than the same sound emitted by a frontal source $\left(0^{\circ}\right)$. This effect, known as directional loudness, is largely accounted for by physical modifications affecting the sound pressure along its way to the listener's ears. The stimulation at each of the two ears is affected to a different extent depending on the source azimuth. Thus the overall loudness resulting from a binaural loudness summation process is affected by the source location [3]. This effect has been mainly reported for high-frequency sounds, for which at-ear pressures are modified because of the perturbation of the sound field by the head. The resulting interaural level difference (ILD) is used for high-frequency localization, above about

Received 13 March 2015, accepted 10 August 2015.
$1500 \mathrm{~Hz}$, but is often considered as negligible below $500 \mathrm{~Hz}$ [4]. However, it has been shown that directional loudness sensitivity (DLS) could be observed at $400 \mathrm{~Hz}$ [2], where there is little acoustic shadow of the head [4]. Moreover, a tendency for the measured DLS values to be slightly higher than model predictions based on the related pressure measurements was reported at $5000 \mathrm{~Hz}$ [5]. Therefore, regardless of whether or not the left and right ear pressures are equal, it may be suspected that directional loudness effects are not caused only by pressure modifications.

In a previous study, an effect of interaural time difference (ITD) on loudness has been shown for low-frequency pure tones at 200 and $400 \mathrm{~Hz}$ [6]. A small but significant increase of loudness with increasing ITD could be observed at a low loudness level (40 phon) but not a moderate one (70 phon). However, it was not clear why this effect only occurred at low loudness level and whether loudness was affected by ITD itself or by higher-level processes. As an example, it has been shown that perceived source distance had an effect on loudness [7]. Further experiments were therefore needed to get insight into the potential cause and extent of this effect. 
As non-negligible ILDs were reported at 200 and $400 \mathrm{~Hz}$ [2], a first experiment was designed to investigate the effects of both ITD and ILD on the loudness of low-frequency pure tones at 40 phon. Stimuli were presented via headphones and lateralized by introducing ITD and ILD. These differences could be congruent and lead to the same side, but could also be opposite and compensate for the lateralization induced by each other [8]. The effects of ITD and ILD on loudness were observed by asking listeners to match dichotic stimuli (obtained by introducing interaural differences) with a diotic one (no interaural difference).

As the ITD effect proved to be significant at low loudness level only [6], a second experiment was designed to investigate the effect of ITD at the hearing threshold. The minimum audible field is measured in free, quasi-free or diffuse sound field using a loudspeaker that is generally placed in front of the listener [9]. Some data for monaural hearing thresholds related to other incidences $\left(45^{\circ}\right.$ and $\left.90^{\circ}\right)$ can be found in the current standards for audiometric tests $[9,10]$. As an example, the data indicate a slight decrease of the monaural hearing threshold when increasing the loudspeaker incidence from $0^{\circ}$ to $90^{\circ}$ at $125 \mathrm{~Hz}$ which suggests that directional effects could possibly occur at this level. However, these reference thresholds were derived from data based on measurements collected in various studies $[11,12]$. In some of these studies, the loudspeaker was placed at $1 \mathrm{~m}$ from the reference point. The variations of the hearing threshold with the source azimuth could then simply be caused by the experimental conditions, i.e., at-ear pressure modifications with the azimuth because of the small distance between the loudspeaker and the listener's ear. In addition to these threshold data for non-frontal incidence, it has also been shown that interaurally outof-phase $250-\mathrm{Hz}$ tones were detected at a $0.9 \mathrm{~dB}$ lower level than when presented in phase [13]. Therefore, it was decided to investigate the effect of ITD at threshold level by measuring hearing thresholds of dichotic stimuli.

\section{Experiment I: loudness of pure tones including ITD and ILD}

The effects of both ITD and ILD on loudness were investigated by matching in loudness dichotic pure tones with a diotic reference of the same frequency.

\subsection{Stimuli}

The two frequencies $(200 \mathrm{~Hz}$ and $400 \mathrm{~Hz}$ ) at which the effect of ITD on loudness has been observed at low loudness level (40 phon) [6] were under study.

Three ITD values $(-772 \mu \mathrm{s}, 0 \mu \mathrm{s},+772 \mu \mathrm{s})$ were selected as $\pm 772 \mu$ s were the ITD values for which the effect on loudness was the highest [6]. These values were obtained from Kuhn's low-frequency model [14], which is valid below $500 \mathrm{~Hz}$ :

$$
\mathrm{ITD}=\frac{3 a}{c_{0}} \sin \theta_{i n c}
$$

where $a=8.75 \mathrm{~cm}$ is the average head radius, $c_{0}=340 \mathrm{~m} \cdot \mathrm{s}^{-1}$ is the speed of sound in the air and $\theta_{i n c}$ is the incidence angle for a virtual sound source in the horizontal plane (respectively $-90^{\circ}, 0^{\circ}$ and $+90^{\circ}$ ). To elicit the impression of a lateralized sound, an ITD was introduced on the basis of Equation (1). ITDs of $\pm 772 \mu$ s are higher than the interaural time/phase difference thresholds at those frequencies and should enable the lateralization of pure tones [15]. A negative sign means that the ITD leads to the left side.

In addition, although ILD is generally considered as small below $500 \mathrm{~Hz}$ [4], it can be seen on HRTF measurements [2] that it is not negligible for an azimuth of $\pm 90^{\circ}$ and can reach up to $2.5 \mathrm{~dB}$ at $200 \mathrm{~Hz}$ and $5 \mathrm{~dB}$ at $400 \mathrm{~Hz}$. Those ILDs consist in a SPL increase on the leading side (respectively $+2.5 \mathrm{~dB}$ and $+5 \mathrm{~dB}$ ), whereas it remains constant on the opposite side. Therefore, an ILD was also introduced between the left and right channels by increasing the level on the leading side only. Five ILD values were selected $(+5 \mathrm{~dB}$ left, $+2.5 \mathrm{~dB}$ left, $0 \mathrm{~dB},+2.5 \mathrm{~dB}$ right, $+5 \mathrm{~dB}$ right). ITD and ILD could be congruent or opposite (e.g. ITD $=+772 \mu$ s and ILD $=+5 \mathrm{~dB}$ left) and should compensate, at least partially, for the lateralization induced by each other in this case [8].

These stimuli were to be matched in loudness to diotic references whose level was fixed at 40 phon by placing the test headphones (Sennheiser HD650, circumaural, open) on a dummy head (Neumann KU100) whose microphones are located at the entrance of the blocked ear canal. Firstly, the sound pressure level was adjusted to $94 \mathrm{~dB}$ SPL at $1000 \mathrm{~Hz}$ on each ear. Starting from this point, the two frequencies under test were subsequently adjusted by following the 40-phon curve defined by ISO 226 [16]. The sound pressure levels corresponding to 40 phon are respectively $53.4 \mathrm{~dB}$ SPL at $200 \mathrm{~Hz}$ and $45.0 \mathrm{~dB}$ SPL at $400 \mathrm{~Hz}$. The duration of each stimulus was $1.6 \mathrm{~s}$. Its onset and offset were smoothed by 100 -ms-long raisedcosine functions. The dummy head also enabled to verify the interaural synchronicity of the diotic pure tones.

\subsection{Procedure}

The point of subjective equality (PSE) was measured for each of the 30 experimental conditions (2 frequencies, 3 ITDs and 5 ILDs) by using a loudness matching task. A two-interval two-alternative forced choice (2I2AFC) paradigm was used with a 1-up-1-down rule [17]. In each trial, a test sound (stimulus including interaural differences) and a reference sound (diotic stimulus at 40 phon) lasting $1.6 \mathrm{~s}$ each were consecutively presented in random order with a $500-\mathrm{ms}$ 
pause in between. The task was to indicate whether the first or the second sound was perceived as louder, regardless of any other difference. The instructions were given both orally and in written form. The subject responded by clicking a button on a MATLAB graphical user interface. The sound pressure level of the test sound was varied from trial to trial depending on the response of the subject. It was lowered by a given amount when the subject judged it to be louder, whereas it was increased by the same amount when the subject judged the reference to be louder. The same level change was applied to both ear signals, i.e., the interaural level difference of the test signal was preserved during the adaptive run. The step size was initially set to $4 \mathrm{~dB}$ and was decreased to $1 \mathrm{~dB}$ after two reversals (a reversal denotes a change in direction in the matching sequence). The starting level of each stimulus was randomly set $10 \mathrm{~dB}$ above or below the diotic reference to provide clearly noticeable loudness differences with at the beginning of the matching process. As a reminder, the level was only increased on the leading side to achieve a non-zero ILD and this offset is thus defined with respect to the opposite side. For a given test sound, the adaptative sequence was ended at the eighth reversal [2]. The arithmetic mean of the levels at the last six reversals was used to derive the PSE of the test sound with respect to its reference. The 30 adaptative sequences related to the experimental conditions were randomly reordered and interleaved on a trial-by-trial basis, providing some random sampling of ITDs, ILDs, frequencies and starting levels. From the subject's point of view, each session appeared as a succession of unrelated paired comparisons of loudness.

The subject sat in an audiometric booth and was asked to place the test headphones comfortably over his ears and to not modify this position once the test had started as realistic changes in the position of such headphones can be audible [18]. The test lasted approximately $1 \mathrm{~h}$ and was preceded by a 3 -min pretest to familiarize the listener with the task and the answering interface. Twenty sound engineering students (Bachelor's and Master's degree) from the University of Brest took part in this experiment and were remunerated for their participation. The subjects (seven women and thirteen men, with ages ranging from 20 to 22 years) had normal hearing thresholds $(\leq 10 \mathrm{~dB}$ HL) based on an audiogram taken in the month preceding this test. None of them had particular experience in laboratory listening tests.

\subsection{Results}

The PSE is presented as the difference between the matched level on the ear that is not affected by ILD and the reference level. As an example, for a test sound with ILD $=0 \mathrm{~dB}$, a negative PSE would indicate that a lower level was required to achieve equal loudness with respect to the reference. Results were analyzed using repeated-measures analysis of variance with frequency (2 levels), ITD (3 levels) and ILD (5 levels) as within-subjects factors.

The effects of ILD $(F(4,76)=174.99 ; p<0.001)$ and $\operatorname{ITD}(F(2,38)=37.51 ; p<0.001)$ were both significant. As there was no significant interaction $(F(8,152)=0.84 ; p=0.573)$ between these two factors, it can be concluded that their effects were additive. The analysis did not highlight any further significant effect or interaction.

For ILD $=0 \mathrm{~dB}$ (Figure 1), the PSE is significantly lower when ITD is $\pm 772 \mu$ s than when it is $0 \mu \mathrm{s}(p<$ 0.001 in both cases according to Fisher's LSD test). Note that the difference is small (around $1 \mathrm{~dB}$ ) but still higher than the just noticeable difference in sound pressure level for such stimuli [19]. This effect is very similar to the one already observed [6]. As the PSE is significantly lower when ITD $= \pm 772 \mu$ s than when ITD $=0 \mu \mathrm{s}$, it can be inferred that, at the same level, the dichotic signals are louder than the diotic signal.

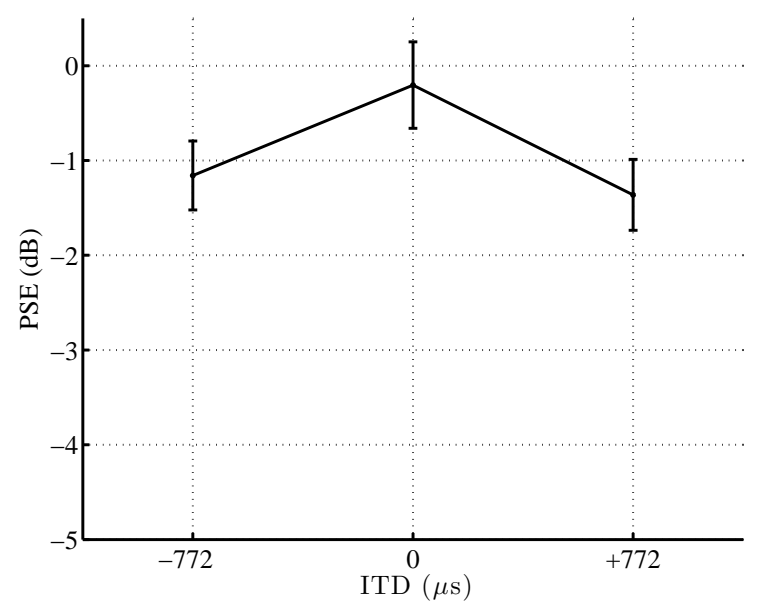

Figure 1. Mean PSE as a function of ITD when ILD is $0 \mathrm{~dB}$, with $95 \%$ confidence intervals.

The ILD was created by adding $2.5 \mathrm{~dB}$ or $5 \mathrm{~dB}$ on the leading side. As expected, the PSE significantly decreases with increasing ILD, namely by around $1.5 \mathrm{~dB}$ if ILD $=+2.5 \mathrm{~dB}$ and by around $3 \mathrm{~dB}$ if $\mathrm{ILD}=+5 \mathrm{~dB}$, whatever the leading side. Figures 2 and 3 depict the PSEs in the same range for each ILD value to highlight this effect. These figures also show that the ITD effect depicted in Figure 1 (ILD $=0 \mathrm{~dB}$ ) applies for the 4 other ILD values. This effect is the same (i.e. a significant decrease of the PSE for ITD $= \pm 772 \mu \mathrm{s}$ ) when ILD and ITD lead to the same side as when they are contrary (i.e. non-significant interaction). As an example, the dashed line on Figure 2 shows that the PSE is the same for ITD $=-772 \mu \mathrm{s}$ and ITD $=+772 \mu$ s although the latter leads to the opposite side of the ILD $(+2.5 \mathrm{~dB}$ left $)$. This is also found for all other non-zero ILDs (Figures 2 and 3 ). 


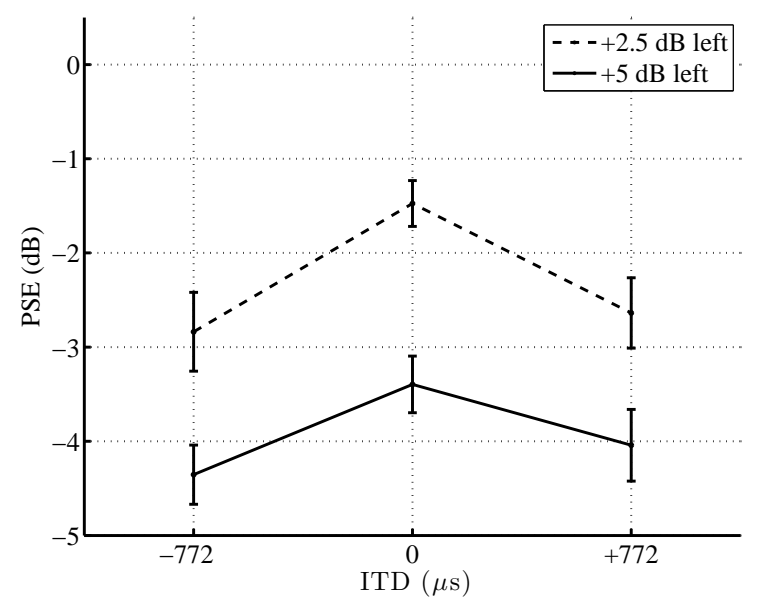

Figure 2. Mean PSE as a function of ITD when ILD leads on the left, with $95 \%$ confidence intervals.

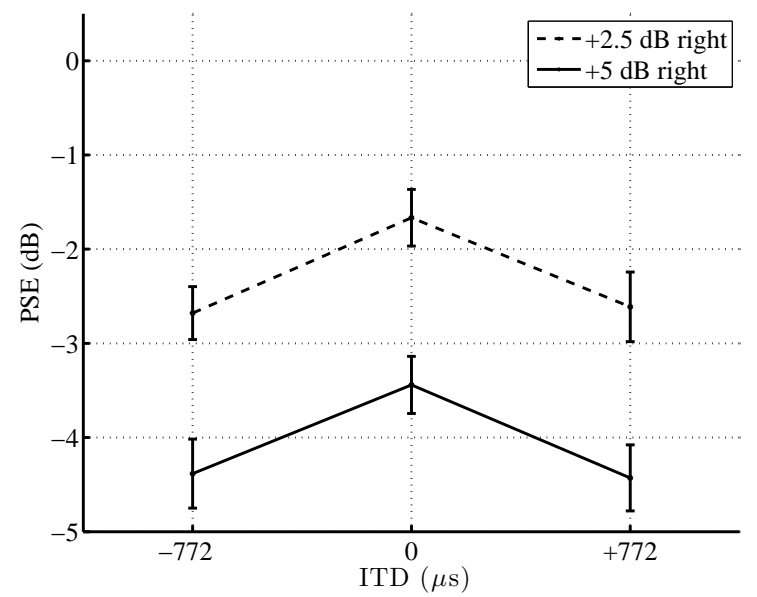

Figure 3. Mean PSE as a function of ITD when ILD leads on the right, with $95 \%$ confidence intervals.

\section{Experiment II: hearing threshold of pure tones including ITD}

The effect of ITD on loudness at absolute threshold level was investigated by measuring the hearing threshold of lateralized pure tones.

\subsection{Stimuli}

Five frequencies were under study in this second experiment :

- 200 and $400 \mathrm{~Hz}$, where ITD had a significant effect on loudness at 40 phon according to a previous study [6] and which was confirmed in Experiment I,

- 125,250 and $500 \mathrm{~Hz}$ which are the closest audiometric octave frequencies.

The frequencies ranged from $125 \mathrm{~Hz}$, where pure tones may already be lateralized on the basis of ITD [15], to $500 \mathrm{~Hz}$, where the acoustic shadow of the head is still small [4]. Thus for all signals used in this experiment, the same pressure at the two ears should appear natural to the listener.
Four ITD values $(0 \mu \mathrm{s},+386 \mu \mathrm{s},+669 \mu \mathrm{s}$ and $+772 \mu \mathrm{s}$ ) were selected in order to lateralize the stimuli on the right. Only one side was chosen since the effect of ITD on loudness was symmetric in a previous study [6] and in Experiment I of the present study. These values were related to virtual incidence angles $\left(0^{\circ},+30^{\circ},+60^{\circ}\right.$ and $\left.+90^{\circ}\right)$ according to Kuhn's low-frequency model [14]. ITD values of $+386 \mu \mathrm{s}$, $+669 \mu \mathrm{s}$ and $+772 \mu \mathrm{s}$ are higher than the interaural time/phase difference thresholds at these frequencies [20] and should enable the lateralization of pure tones. The headphones (Sennheiser HD650, circumaural, open) were calibrated the same way as in Experiment I. Firstly, the sound pressure level was adjusted to $94 \mathrm{~dB}$ SPL at $1000 \mathrm{~Hz}$ on each ear. Starting from this point, the five frequencies under test were subsequently adjusted by following the hearing threshold curve defined by ISO 226 [16]. Table I indicates these standard threshold values. The duration of each stimulus was $1.6 \mathrm{~s}$.

Table I. Sound pressure level at the hearing threshold according to the frequency.

\begin{tabular}{|c|c|}
\hline$f(\mathrm{~Hz})$ & $L(\mathrm{~dB}$ SPL $)$ \\
\hline 125 & 22.0 \\
200 & 14.5 \\
250 & 11.0 \\
400 & 6.0 \\
500 & 4.0 \\
\hline
\end{tabular}

\subsection{Procedure}

Hearing thresholds were measured for the 20 experimental conditions ( 5 frequencies, 4 ITDs) by using a custom procedure [21] based on ANSI S3.21 [22]. Each threshold measurement was carried out by presenting the stimulus (pure tone) at a level set $50 \mathrm{~dB}$ above the standardized hearing threshold (Table I). A one-interval two-alternative forced choice (1I2AFC) paradigm was used with a 1-up-2-down rule. After each stimulus presentation, the listener was asked whether he had perceived the sound or not. Similarly to Experiment I, instructions were given both orally and in written form and the subject responded by clicking a button on a MATLAB graphical user interface. The level of the stimulus was lowered in the case of a positive answer, whereas it was increased in the case of a negative answer. The current procedure consisted of three bracketing series, each providing progressively smaller step sizes to finally result in threshold responses with $1-\mathrm{dB}$ resolution. The initial bracketing series provided step sizes of up $5 \mathrm{~dB}$, down $10 \mathrm{~dB}$ to quickly bracket the threshold level to within $10 \mathrm{~dB}$. Intermediate bracketing series provided step sizes of up $2 \mathrm{~dB}$, down $5 \mathrm{~dB}$ and was entered after two reversals (a reversal denotes a change in direction 
in the measurement process). The final bracketing series, in which the hearing threshold was measured, was reached after two more reversals (four reversals in all) and provided step sizes of up $1 \mathrm{~dB}$, and down $2 \mathrm{~dB}$. The level at which a positive answer occurred was defined as the threshold if at least one of the two preceding positive answers within the final series occurred at the same level. Threshold value could not be reached this way before the eighth reversal.

The listener sat in an audiometric booth and was asked to place the HD650 headphones comfortably over his ears and to not modify this position once the test had started. It has also been shown that realistic changes in such headphones position could lead to significant differences in hearing threshold measurements [21]. In order to take into account test-retest variability [22], all subjects carried out the experiment twice, on two different days. The test lasted approximately $45 \mathrm{~min}$ and was preceded by a 3-min pretest to familiarize the listener with the task and the answering interface. Fifteen sound engineering students (Bachelor's and Master's degree) from the University of Brest took part in this experiment and were remunerated for their participation. The subjects (five women and ten men, with ages ranging from 20 to 22 years) had normal hearing thresholds $(\leq 10 \mathrm{~dB} \mathrm{HL})$ based on an audiogram taken in the month preceding this test. None of them had particular experience in laboratory listening tests.

\subsection{Results}

For each listener at each frequency, the two measurements with zero ITD were averaged to define a sensation level reference (0 dB SL). All threshold measurements related to a given listener at a given frequency (4 ITDS and 2 repetitions) were subsequently shifted according to its respective reference, in order to take into account individual threshold differences. The thresholds were analyzed using repeatedmeasures analysis of variance with test repetition (2 levels), frequency (5 levels) and ITD (4 levels) as within-subjects factors.

This analysis did not highlight any significant effect or interaction among the factors under test. In particular, no simple effect $(F(3,42)=0.66 ; p=0.581)$ or interaction involving ITD proved to be significant. As can be noted from Figure 4, the hearing threshold was statistically equivalent for all ITD values under test.

\section{Discussion}

The results of Experiment I indicate significant effects of both ITD and ILD on loudness for low-frequency pure tones, but no interaction between these two factors. The loudness of the tones were the same whether their ILD led to the same side as their ITD or to the

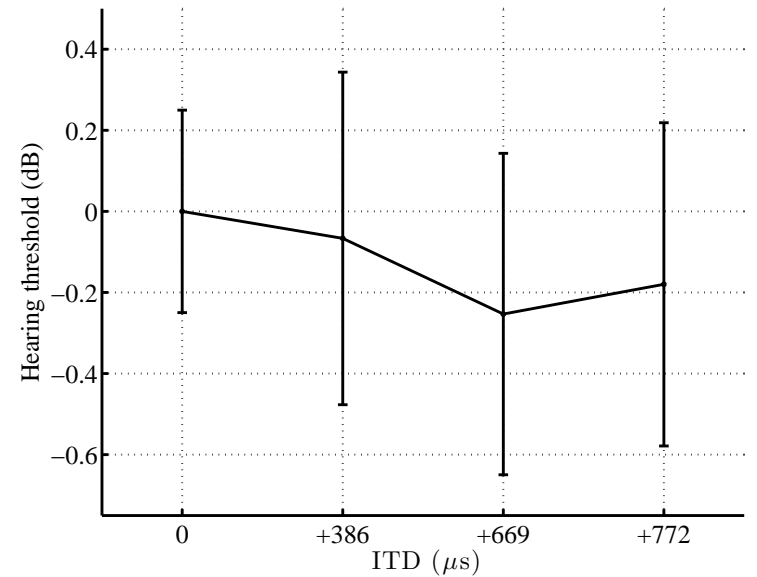

Figure 4. Mean hearing threshold as a function of ITD, with $95 \%$ confidence intervals.

opposite one, which would compensate for the lateralization induced by each other in the latter case [8]. This does not support one of the two hypotheses developed to explain the ITD effect observed in a previous paper [6]. This hypothesis stated that the effect of ITD on loudness would be due to "high-level" processes as the lateralization of the source would have been taken into account in the loudness process. Such effects were reported about the influence of perceived source distance on loudness [7]. On the other hand, these results confirm the effect of ITD on loudness [6] and are consistent with the alternative hypothesis that ITD could help separate the signal from internal noise exhibiting a small positive correlation [13, 23]. This hypothesis relies on the assumption that the signal is perceived as a separate entity from the background noise and that its loudness might be enhanced if ITD provides a better separability [24]. This could explain why loudness is affected by interaural phase/time difference at a low loudness level (40 phon) independently of the ILD effect.

Following this hypothesis further, one would have expected the ITD effect to be observed at even lower levels. The hearing threshold can be considered as the starting point of measurable loudness, at which the loudness is very small [25] and considered to be a fixed value [26]. However, the results of Experiment II did not highlight any significant effect of the ITD values under test $(+386 \mu \mathrm{s},+669 \mu \mathrm{s}$ and $+772 \mu \mathrm{s})$ at the absolute threshold. It might be primarily inferred that ITD has no effect on loudness at very small levels, but the lack of significance could be also due to the fact that the ITD effect is hardly measurable in this case. As an example, a difference of $1 \mathrm{~dB}$ in the PSEs was observed at 40 phon as it is superior to the differential threshold which increases with decreasing loudness level [19]. However, it has been reported for $250-\mathrm{Hz}$ tones that reversing the interaural phase lowers the hearing threshold by $0.9 \mathrm{~dB}$ [13]. For the ITDs and frequencies under test, the corresponding inter- 
aural phase differences are always less than $\pi$ which could imply an even lower effect. In addition, the sensitivity to the frequencies under test may vary from one listener to another because of the fine structure of the hearing threshold [27]. A given frequency could possibly lie within a range of minimum or maximum sensitivity, so that the ITD effect would not be the same and that the variability of the threshold measurements would be increased.

In summary, the results of this study and of a previous one [6] indicate both a significant effect of the ITD at a low loudness level (40 phon). At this level, ITD could have enabled a better separability from the internal noise and enhanced the loudness of the tone. Similarly, loudness increases with increasing interaural phase differences were reported up to $40 \mathrm{~dB}$ above the masked threshold [28]. However, several other studies did not show a significant effect over such a wide range $[29,30]$. At higher loudness levels, the internal noise should be all the more negligible and such an effect was not observed at 70 phon [6]. The ITD effect was also not significant at the hearing threshold as it might be hardly observable at very small loudness levels.

\section{Conclusion}

The results of the present study show a significant effect of ITD on the loudness of low-frequency pure tones presented at a low loudness level (40 phon) independently of the ILD. As this effect applied even when ILD led to the opposite side of the ITD, it can be inferred that the effect of ITD on loudness is not related to the lateralization. This supports the assumption that directional loudness is rather due to the localization cues (namely ITD and ILD) than to the localization process itself. However, no significant effect of ITD was found for pure tones at the hearing threshold. A possible ITD effect on loudness at very low levels might therefore be hardly observable.

\section{Acknowledgement}

The authors wish to thank the staff and students from the "Image \& Son" department of the University of Brest for participating in this experiment.

\section{References}

[1] D. W. Robinson, L. S. Whittle: The loudness of directional sound fields. Acta Acustica united with Acustica 10 (1960) 74-80.

[2] V. P. Sivonen, W. Ellermeier: Directional loudness in an anechoic sound field, head-related transfer functions, and binaural summation. The Journal of the Acoustical Society of America 119 (2006) 2965-2980.

[3] V. P. Sivonen, W. Ellermeier: Binaural loudness. - In: Loudness. M. Florentine, A. N. Popper, R. R. Fay (eds.). Springer, New York City, NY, USA, 2011, 169-179.

[4] B. C. J. Moore: An introduction to the psychology of hearing. Sixth edition. Brill, Leiden, The Netherlands, 2012, 245-250.
[5] B. C. J. Moore, B. R. Glasberg: Modeling binaural loudness. The Journal of the Acoustical Society of America 121 (2007) 1604-1612.

[6] V. Koehl, M. Paquier: Loudness of low-frequency pure tones lateralized by interaural time differences. The Journal of the Acoustical Society of America 137 (2015) 1040-1043.

[7] P. Zahorik, D. S. Brungart, A. W. Bronkhorst: Auditory distance perception in humans: A summary of past and present research. Acta Acustica united with Acustica 91 (2005) 409420.

[8] G. G. Harris: Binaural interactions of impulsive stimuli and pure tones. The Journal of the Acoustical Society of America 32 (1960) 685-692.

[9] ISO 8253-2: Acoustics - Audiometric test methods - Part 2: Sound field audiometry with pure-tone and narrow-band test signals. International Organization for Standardization, Geneva, Switzerland, 2009.

[10] ANSI S3.6: Specification for audiometers. American National Standards Institute, New York City, NY, USA, 2010.

[11] E. A. G. Shaw: Transformation of sound pressure level from the free field to the eardrum in the horizontal plane. The Journal of the Acoustical society of America 56 (1974) 18481861.

[12] E. A. G. Shaw, M. M. Vaillancourt: Transformation of sound pressure level from the free field to the eardrum in the horizontal plane presented in numerical form. The Journal of the Acoustical society of America 78 (1985) 1120-1123.

[13] K. J. Diercks, L. A. Jeffress: Interaural phase and the absolute threshold for tone. The Journal of the Acoustical Society of America 34 (1962) 981-984.

[14] R. G. Klumpp, H. R. Eady: Model for the interaural time differences in the azimuthal plane. The Journal of the Acoustical Society of America 62 (1977) 157-167.

[15] P. X. Zhang, W. M. Hartmann: Lateralization of sine tonesinteraural time vs phase. The Journal of the Acoustical Society of America 120 (2006) 3471-3474.

[16] ISO 226: Acoustics - Normal equal-loudness-level. International Organization for Standardization, Geneva, Switzerland, 2003.

[17] H. Levitt: Transformed up-down methods in psychoacoustics. The Journal of the Acoustical society of America 49 (1971) $467-477$.

[18] M. Paquier, V. Koehl: Discriminability of the placement of supra-aural and circumaural headphones. Applied Acoustics 93 (2015) 130-139.

[19] H. Fletcher: Minimum perceptible changes in frequency and sound pressure level. - In: Speech and Hearing in Communication. Van Nostrand, Princeton, NJ, USA, 1953, 144-152.

[20] R. G. Klumpp, H. R. Eady: Some measurements of interaural time difference thresholds. The Journal of the Acoustical Society of America 28 (1956) 859-860.

[21] M. Paquier, V. Koehl, B. Jantzem: Influence of headphone position in pure-tone audiometry. Proceedings of Acoustics 2012 Conference, Nantes, France, 2012, 3925-3930.

[22] ANSI S3.21: Methods for manual pure-tone threshold audiometry. American National Standards Institute, New York City, NY, USA, 2009.

[23] W. A. Yost: The masking-level difference and overall masker level: Restating the internal noise hypothesis. The Journal of the Acoustical Society of America 83 (1988) 1517-1521.

[24] B. A. Edmonds, J. F. Culling: Interaural correlation and the binaural summation of loudness. The Journal of the Acoustical Society of America 125 (2009) 3865-3870.

[25] B. C. J. Moore, B. R. Glasberg, B. R, T. Baer: A model for the prediction of thresholds, loudness, and partial loudness. Journal of the Audio Engineering Society 45 (1997) 224-240.

[26] B. C. J. Moore: Development and current status of the "cambridge" loudness models. Trends in Hearing 18 (2014) 1-19.

[27] M. Mauermann, G. R. Long, B. Kollmeier: Fine structure of hearing threshold and loudness perception. The Journal of the Acoustical Society of America 116 (2004) 1066-1080.

[28] E. Zwicker, G. B. Henning: On the effect of interaural phase differences on loudness. Hearing research 53 (1991) 141-152.

[29] T. H. Townsend, D. P. Goldstein: Suprathreshold binaural unmasking. The Journal of the Acoustical Society of America 51 (1972) 621-624.

[30] J. L. Verhey, S. J. Heise: Suprathreshold perception of tonal components in noise under conditions of masking release. Acta Acustica united with Acustica 98 (2012) 451-460. 